\title{
Segurança e efetividade no tratamento do pterígio usando infiltração de 5-fluoruracila no intraoperatório
}

\author{
Safety and efficacy of intraoperative 5-fluorouracil infiltration \\ in pterygium treatment
}

\author{
Vanessa Grandi Valezi ${ }^{1}$ \\ Silvana Artioli Schellini ${ }^{2}$ \\ Magda Massae Hata Viveiros ${ }^{3}$ \\ Carlos Roberto Padovani ${ }^{4}$
}

\begin{tabular}{l} 
RESUMO \\
\hline Objetivo: Avaliar a segurança e a efetividade do uso do 5-fluoruracila \\
(5-FU) como tratamento adjuvante do pterígio, aplicado sob a forma de \\
infiltração subconjuntival, no período intraoperatório. Métodos: Foram \\
avaliados prospectivamente 125 indivíduos (125 olhos) portadores de \\
pterígio. Os indivíduos foram operados segundo a técnica de retalho de \\
deslizamento e receberam, ao final do procedimento, injeção subcon- \\
juntival de 0,2 mL de 5-FU (25 mg/mL). Foram anotados os dados do \\
paciente como idade, sexo, profissão, características da lesão (primário \\
ou recidivado, tamanho, carnoso ou involutivo) e feito seguimento pós- \\
operatório, aos 7, 21, 60 e 180 dias. Os dados foram submetidos à \\
avaliação estatística. Resultados: Não foram observados casos de com- \\
plicação decorrente do uso do 5-FU em injeção no intraoperatório do \\
pterígio. A taxa de recidiva geral observada aos 180 dias de pós-operatório \\
foi de 35,8\%, sendo de 35,7\% para os pterígios primários e de 36,4\% para \\
os recidivados. Conclusão: A aplicação do 5-FU no período intra- \\
operatório sob a forma de infiltração subconjuntival é segura. Entretan- \\
to, ainda resulta em altas taxas de recidiva e novos estudos devem ser \\
realizados a fim de conhecer a concentração/dose ideal que permitirá \\
menores chances de recidiva da lesão.
\end{tabular}

Descritores: Pterígio; Fluoruracila; Mitomicina/administração \& dosagem; Quimioterapia adjuvante; Antimitóticos; Avaliação de eficácia-efetividade de intervenções; Cuidados pré-operatórios; Recidiva/prevenção \& controle

\section{INTRODUÇÃO}

Residente do Departamento de Oftalmologia/Otorrinolaringologia/Cabeça e Pescoço da Faculdade de Medicina de Botucatu da Universidade Estadual Paulista UNESP - Botucatu (SP) - Brasil.

${ }^{2}$ Livre-Docente do Departamento de Oftalmologia/Otorrinolaringologia/Cabeça e Pescoço da Faculdade de Medicina de Botucatu da UNESP - Botucatu (SP) - Brasil.

${ }^{3}$ Pós-Graduanda nível Doutorado, Faculdade de Medicina de Botucatu da UNESP - Botucatu (SP) - Brasil.

${ }^{4}$ Professor Titular do Departamento de Bioestatística, Instituto de Biociências, Faculdade de Medicina de Botucatu da UNESP - Botucatu (SP) - Brasil.

Endereço para correspondência: Silvana Artioli Schellini. DEP. OFT/ORL/CCP - Faculdade de Medicina de Botucatu - UNESP - Botucatu (SP) CEP 18618-000

E-mail: sartioli@fmb.unesp.br

Recebido para publicação em 07.01.2007

Última versão recebida em 24.02.2008

Aprovação em 29.01.2009

\begin{tabular}{l}
\hline INTRODUÇão \\
\hline Pterígio é uma proliferação fibrovascular triangular da conjuntiva que \\
cresce em direção à córnea, infiltrando sua superfície. Sua patogênese ainda \\
não completamente esclarecida, associada a uma variável taxa de recidiva \\
pós-operatória, constitui um desafio para o médico oftalmologista no que se \\
refere à escolha da melhor opção terapêutica ${ }^{(1)}$. \\
Inúmeras técnicas cirúrgicas já foram testadas e, apesar da maioria destas \\
serem relativamente simples, é comum a ocorrência de recidivas, levando à \\
necessidade do uso de drogas coadjuvantes. \\
A recidiva do pterígio é atribuída à proliferação de fibroblastos, tendo sido \\
introduzidas drogas antimitóticas com o intuito de prevenir o novo crescimento \\
da lesão, dentre elas o thio-tepa, a mitomicina C e o 5-fluoruracila (5-FU). O \\
uso do thio-tepa foi feito na década de 60 e provocava alterações oculares \\
transitórias e até mesmo déficit visual permanente ${ }^{(2-4)}$. A mitomicina C surgiu
\end{tabular}


na década de 80 como uma droga antimitótica promissora no tratamento coadjuvante do pterígio ${ }^{(5-7)}$, com diminuição da taxa de recidiva do pterígio primário e recidivado, mas havendo relatos de diversas complicações (glaucoma secundário, edema e perfuração corneana, corectopia, irite, catarata, calcificação escleral, fotofobia, dor incapacitante) ${ }^{(8-12)}$.

Buscando-se uma nova droga que substituísse a mitomicina $\mathrm{C}$ e que pudesse evitar seus efeitos deletérios, pesquisas foram desenvolvidas com o 5-FU, uma droga que, assim como a mitomicina $\mathrm{C}$, possui ação antifibroblástica pela inibição do DNA, atuando, porém, por mecanismos diferentes, proporcionando citotoxicidade e inibição da proliferação de fibroblastos da cápsula de Tenon menores ${ }^{(13)}$, o que o torna o 5-FU uma droga mais segura.

Os estudos realizados utilizando-se o 5-FU foram feitos de diversas maneiras, entre elas, testando-se o 5-FU tópico, aplicado no intraoperatório, observando-se redução da taxa de recidiva e sem as complicações observadas com a mitomicina $\mathrm{C}^{(14)}$.

O 5-FU também foi testado em aplicação sob a forma de infiltração intralesional, na cabeça do pterígio, no período préoperatório, resultando em índice de recidiva superior ao encontrado com o uso tópico intraoperatório ${ }^{(15)}$.

O presente estudo foi desenvolvido com o objetivo avaliar a segurança e a efetividade do 5-FU em prevenir as recidivas da lesão, quando aplicado sob a forma de infiltração subconjuntival, no período intraoperatório.

\section{MÉTODOS}

Este foi um estudo prospectivo, com intervenção, e incluiu 125 olhos de 125 portadores de pterígios primários e recidivados, atendidos consecutivamente no Hospital das Clínicas da UNESP, Botucatu - SP, no ano de 2005. O protocolo deste estudo foi aprovado pelo Comitê de Ética em Pesquisa em Seres Humanos da Faculdade de Medicina de Botucatu.

A classificação dos pterígios foi feita por um mesmo examinador, de acordo com a padronização usada no serviço, baseada na observação clínica das seguintes características:

1. Primário ou recidivado;

2. Aspecto da proliferação fibrovascular:

a) Involutivo: aspecto atrófico, visibilização dos vasos episclerais no limbo;

b) Carnoso: aspecto espessado, impossibilitando observar vasos episclerais.

3. De acordo com a extensão sobre a superfície corneana:

a) Grau 1: atinge o limbo em até $2 \mathrm{~mm}$;

b) Grau 2: atinge a borda pupilar;

c) Grau 3: ultrapassa a borda pupilar, atingindo o eixo visual.

Todas as cirurgias foram realizadas por dois cirurgiões e a mesma equipe de auxiliares, empregando-se a técnica do retalho conjuntival de deslizamento, iniciando-se pela anestesia tópica com cloridrato de proximetacaína e subconjuntival com lidocaína $2 \%$ com vasoconstritor. Procedeu-se a delami- nação corneana com bisturi lâmina 15 , exérese da cabeça do pterígio e ressecção da Tenon adjacente com tesoura de conjuntiva. Foi realizado deslizamento de retalhos superior e inferior para fechamento da conjuntiva próxima, na região do limbo, usando fio trançado absorvível sintético 7-0 (Vicryl Ethicon), em dois pontos separados, recobrindo-se a esclera desnuda pela remoção da lesão.

Foi, então, feita em todos os pacientes injeção subconjuntival de 0,2 $\mathrm{mL}$ de 5-FU (Roche; $25 \mathrm{mg} / \mathrm{mL}$ ), localizada na região remanescente do corpo do pterígio, adjacente ao lago lacrimal, usando uma seringa de $1 \mathrm{~mL}$ e agulha de insulina $(13,0 \times 4,5 \mathrm{G})$.

Ao final do procedimento, foram aplicados colírio com associação de cloranfenicol e dexametasona e pomada com associação de ácido retinóico, cloranfenicol e vitamina A. O curativo oclusivo foi mantido por 24 horas e retirado pelo próprio paciente, no domicílio. Durante o pós-operatório foi prescrito colírio com associação de cloranfenicol e dexametasona de $4 / 4$ horas por 7 dias e de $6 / 6$ horas até 21 dias, quando então, a medicação era suspensa.

Após a cirurgia, os pacientes foram avaliados no $7^{\circ}, 21^{\circ}$, $60^{\circ}$ e $180^{\circ}$ dias, observando-se as queixas e, ao exame biomicroscópico, o aspecto da região operada (em relação à presença de hiperemia, ou outras possíveis alterações, por avaliação qualitativa subjetiva) e a presença de complicações. Considerou-se como recidiva o reaparecimento de proliferação fibrovascular, avançando sobre a córnea.

Os dados relativos à distribuição da recidiva do pterígio segundo sexo, faixa etária e tipo de lesão nos períodos de avaliação foram submetidos à análise estatística descritiva pelo método de intervalo de confiança para a proporção de ocorrência ${ }^{(16)}$. Para compensação dos casos que deixaram o seguimento antes do período de 180 dias, as comparações foram feitas usando médias ponderadas.

\section{RESULTADOS}

Durante o período pós-operatório nenhum paciente desenvolveu complicações que pudessem ser imputadas ao medicamento usado.

Dos 125 pacientes que iniciaram o seguimento, 66 chegaram até o final das avaliações planejadas.

Com relação à evolução pós-operatória, observou-se que as recidivas não foram precoces, tendo sido detectadas, em geral, após os 60 dias da cirurgia.

Houve uma taxa de recidiva geral de $35,8 \pm 11,5 \%$ aos 180 dias de pós-operatório $(\mathrm{p} \leq 0,05)$. A avaliação da recidiva segundo as faixas etárias mostrou taxa de $44,4 \pm 32,5 \%$ na faixa etária de 21-30 anos, de 37,5 $\pm 23,7 \%$ na faixa etária de 31-40 anos, de 35,3 $\pm 22,7 \%$ na faixa etária de 41-50 anos e, de 32,0 $\pm 18,3 \%$ na faixa etária $>$ de 50 anos $(\mathrm{p} \leq 0,05)$ (Tabela 1$)$.

A taxa de recidiva foi semelhante para ambos os sexos, observando-se $37,5 \pm 19,4 \%$ para os homens e de $34,9 \pm 14,2 \%$ para as mulheres $(\mathrm{p} \leq 0,05)$ (Tabela 2$)$. 
Finalmente, a taxa de recidiva foi também semelhante quanto ao tipo de lesão, sendo de $35,7 \pm 12,5 \%$ para os pterígios primários $(\mathrm{p} \leq 0,05)$ e $36,4 \pm 28,4 \%$, para os recidivados $(\mathrm{p} \leq 0,05)$ (Tabela 3$)$.

\section{DISCUSSÃO}

A aplicação do 5-FU da forma como foi feita, em injeção no período intraoperatório foi segura, não tendo sido identificado nenhum paciente com complicações advindas do uso da droga.

No presente estudo, é clara a observação de que as recidivas, em geral, não ocorrem nos primeiros dias de pós-operatório, o que torna necessário se estabelecer longo período de seguimento após a cirurgia, a fim de se detectar os reais índices de recidiva da lesão. Esta observação concorda com a literatura que recomenda tempo médio de observação dos pacientes para detecção da recidiva deve ser, no mínimo, 5 a 6 meses ${ }^{(17)}$.
Apesar de muitos pacientes terem abandonado o seguimento antes do período programado, poder-se-ia interpretar estes resultados como positivos, uma vez que os pacientes que estão bem possuem a tendência de negligenciar o acompanhamento. $\mathrm{O}$ uso da média ponderada nos auxiliou a contornar este problema que é comum nos estudos prospectivos.

As taxas de recidiva não diferiram significativamente quanto ao sexo, idade ou tipo de lesão, o mesmo tendo sido observado em outro estudo ${ }^{(17)}$, apesar de ser clássico que os jovens possuem mais chance de ter novo crescimento da lesão após remoção.

Os resultados do presente estudo são dificilmente comparáveis com a literatura, uma vez que há diferenças entre técnicas, meio de utilização da droga, concentração e dose utilizada, além do que o 5-FU como droga adjuvante no tratamento do pterígio ainda é pouco empregado.

Há relato do uso da técnica de esclera nua e aplicação tópica intraoperatória de 5-FU, na concentração de $10 \mathrm{mg} / \mathrm{mL}$, com

\begin{tabular}{|c|c|c|c|c|c|c|c|c|}
\hline \multirow[b]{2}{*}{ Idade } & \multicolumn{2}{|c|}{7 dias } & \multicolumn{2}{|c|}{21 dias } & \multicolumn{2}{|c|}{60 dias } & \multicolumn{2}{|c|}{180 dias } \\
\hline & Recidiva & Sem recidiva & Recidiva & Sem recidiva & Recidiva & Sem recidiva & Recidiva & Sem recidiva \\
\hline$\leq 20$ & - & - & - & - & - & - & - & - \\
\hline $21-30$ & 1 & 18 & 0 & 16 & 4 & 10 & 4 & 5 \\
\hline $31-40$ & 0 & 26 & 0 & 23 & 3 & 16 & 6 & 10 \\
\hline $41-50$ & 0 & 38 & 1 & 34 & 5 & 28 & 6 & 11 \\
\hline$>50$ & 0 & 42 & 0 & 37 & 5 & 28 & 8 & 17 \\
\hline Total & 1 & 124 & 1 & 110 & 17 & 80 & 24 & 43 \\
\hline $\begin{array}{l}\text { Taxa de } \\
21-30 \text { ar } \\
31-40 \text { ar } \\
41-50 \text { ar } \\
>50 \text { an }\end{array}$ & $\begin{array}{ll}\text { por faixa et } \\
, 4 \% & \text { Taxa } \\
, 5 \% & \text { Taxa } \\
, 3 \% & \text { Taxa } \\
\% & \text { Taxa }\end{array}$ & $\begin{array}{l}\text { avaliada aos } 180 \\
\text { ima: } 12,0 \leq 21-30 \\
\text { ima: } 13,8 \leq 31-40 \\
\text { ima: } 12,6 \leq 41-50 \\
\text { ima: } 13,7 \leq>50 \text { an }\end{array}$ & $\begin{array}{l}\mathrm{s}: \\
\mathrm{s} \leq \text { Taxa máx } \\
\mathrm{s} \leq \text { Taxa máx } \\
\mathrm{s} \leq \text { Taxa máx } \\
\leq \text { Taxa máxim }\end{array}$ & $\begin{array}{l}\text { ima: } 77,0 \% \\
\text { ima: } 61,2 \% \\
\text { ima: } 58,0 \% \\
\text { la: } 50,3 \%\end{array}$ & & & & \\
\hline
\end{tabular}

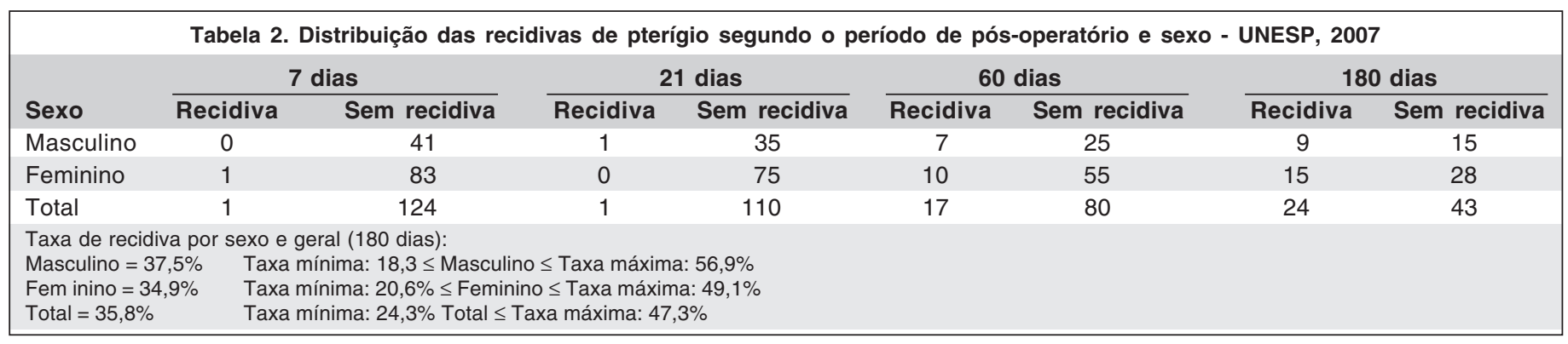

\begin{tabular}{|c|c|c|c|c|c|c|c|c|}
\hline \multirow[b]{2}{*}{ Tipo } & \multicolumn{2}{|c|}{7 dias } & \multicolumn{2}{|c|}{21 dias } & \multicolumn{2}{|c|}{60 dias } & \multicolumn{2}{|c|}{180 dias } \\
\hline & Recidiva & Sem recidiva & Recidiva & Sem recidiva & Recidiva & Sem recidiva & Recidiva & Sem recidiva \\
\hline Primário & 1 & 100 & 1 & 87 & 13 & 62 & 20 & 36 \\
\hline Recidivado & 0 & 24 & 0 & 23 & 4 & 18 & 4 & 7 \\
\hline Total & 1 & 124 & 1 & 110 & 17 & 80 & 24 & 43 \\
\hline $\begin{array}{l}\text { Taxa de recidi } \\
\text { Pterígio primá } \\
\text { Pterígio recidi }\end{array}$ & $\begin{array}{l}\text { la por tipo: } \\
\text { io = 35,7\% } \\
\text { ado }=36,4 \%\end{array}$ & $\begin{array}{l}\text { Taxa mínima: } 23, \\
\text { Taxa mínima: } 7.9\end{array}$ & $\begin{array}{l}\leq \text { Primário } \leq \\
\leq \text { Recidivado }\end{array}$ & $\begin{array}{l}\text { axa máxima: } 48,3 \% \\
\text { Taxa máxima: } 64,8\end{array}$ & & & & \\
\hline
\end{tabular}


taxa de recidiva de $60 \%$, tendo aqueles autores considerado a droga como inefetiva ${ }^{(18)}$. Entretanto, sabe-se que a técnica de esclera nua oferece resultados pobres, além do que o 5-FU é uma droga que, assim como a mitomicina $\mathrm{C}$, é dose e tempo dependente $^{(18)}$, havendo sido constatado efeito semelhante à mitomicina $\mathrm{C}$ quando empregada na dose de 25 a $50 \mathrm{mg} / \mathrm{mL}^{(19)}$.

Comparando a mitomicina $\mathrm{C}$ com o 5 -FU em pterígios primários, houve diferença na taxa de recidiva estatisticamente significativa somente com a mitomicina $\mathrm{C}$ usada no intraoperatório (17\%) comparativamente com seus controles (42\%), com $39 \%$ de recidiva com o 5-FU no intraoperatório e $30 \%$ com 5-FU via subconjuntival em 3 doses, no pós-operatório, resultados considerados semelhantes estatisticamente ${ }^{(20)}$.

Quando a comparação feita foi entre o 5-FU com a betaterapia em pterígios primários, os índices de recidiva foram de $11 \%$ com o antimitótico e $6,5 \%$ com a betaterapia, porém sem diferença estatisticamente significativa e com poucas complicações relatadas com ambos ${ }^{(21)}$.

Desde 1999, o 5-FU tem sido alvo de estudos na Faculdade de Medicina de Botucatu. A utilização do 5-FU para o tratamento coadjuvante do pterígio foi feita no intraoperatório, com aplicação tópica usando cotonete embebido na droga, na concentração de $25 \mathrm{mg} / \mathrm{mL}$, durante 5 minutos, associado à técnica de retalho de deslizamento, com índice de recidiva de 3,5\%, tendo sido a última avaliação feita após 60 dias da operação ${ }^{(14)}$.

Baseados na sugestão da literatura que preconizava a utilização da mitomicina $\mathrm{C}$ em injeção subconjuntival 30 dias ou 3 semanas antes do procedimento cirúrgico ${ }^{(22)}$, optou-se então, pela aplicação preoperatória de $0,2 \mathrm{ml}$ de 5 -FU $(25 \mathrm{mg} / \mathrm{mL})$, intralesional, na cabeça do pterígio, $30(\mathrm{G} 1)$ ou $10(\mathrm{G} 2)$ dias antes da cirurgia. Os índices de recidiva foram muito altos, com $52,9 \%$ no grupo G1 e $27,3 \%$ no G2 quando se tratava de pterígios primários e de $75 \%$ no G1 e $47,1 \%$ no G2 quando os pterígios eram recidivados, sendo que não ocorreram complicações decorrentes da infiltração da droga ${ }^{(15)}$.

E por fim, levando-se em conta que as citocinas serão liberadas após o procedimento cirúrgico, o emprego da droga no intraoperatório nos pareceu ser o mais adequado, o que resultou no presente estudo que mostra que a taxa de recidiva utilizando a técnica de remoção da lesão por retalho de deslizamento e a infiltração de 5-FU no intraoperatório na concentração de $25 \mathrm{mg} / \mathrm{mL}$ e na dose de $0,2 \mathrm{~mL}$, foi de $35,8 \%$.

Em outro estudo, feito com indivíduos operados com técnica cirúrgica semelhante a aqui empregada e usando o 5-FU $25 \mathrm{mg} / \mathrm{mL}$ no intraoperatório de pterígios primários, na forma de aplicação com esponjas sobre a região da esclera desnuda e também sob a conjuntiva e Tenon remanescentes no setor nasal, durante 5 minutos, seguindo-se da aplicação subconjuntival semanal nos indivíduos que passaram a apresentar sinais de recidiva, os autores observaram taxa de recidiva de $14 \%$ e recomendam o emprego desta droga por terem considerado que a mesma é efetiva e segura ${ }^{(17)}$.

Portanto, parece claro que a concentração do 5-FU para impedir a proliferação dos fibroblastos da cápsula de Tenon, ou seja, para impedir a recidiva do pterígio é que precisa ser melhor conhecida e que, sem dúvida, está acima da que foi por nós utilizada.

Similarmente, observando as aplicações de 5-FU feitas para tratamento do glaucoma, a concentração de $50 \mathrm{mg} / \mathrm{mL}$ ainda é considerada segura ${ }^{(23)}$. Outra sugestão para estudos futuros seria o emprego em infiltrações no pós-operatório, para os casos em que se detecte indícios de recidiva da lesão como sugerido por outros ${ }^{(17)}$.

\section{CONCLUSÃO}

A aplicação de $25 \mathrm{mg} / \mathrm{mL}$ do 5-FU no período intraoperatório sob a forma de infiltração subconjuntival é segura. Entretanto, utilizando a droga desta forma e seguindo a técnica cirúrgica aqui empregada, ainda resulta em altas taxas de recidiva. Novos estudos devem ser realizados a fim de conhecer a concentração/dose ideal que permitirá menores chances de recidiva da lesão.

\section{ABSTRACT}

Purpose: To investigate the safety and efficacy of intraoperative infiltration of 5-fluorouracil (5-FU) as an adjuvant drug in pterygium treatment. Methods: Of 125 consecutive patients, 125 eyes with primary and recurrent pterygium underwent pterygium excision with intraoperative 5 -FU $(25 \mathrm{mg} / \mathrm{mL})$ infiltration. The superior and inferior conjunctiva was approximated to cover the scleral bed and $0.2 \mathrm{~mL} 5$-FU was injected at the end of the surgical procedure. The gender, occupation, pterygium characteristics and the follow-up at 7 , 21, 60 e 180 days after surgery were evaluated and the data were statistically analyzed. Results: With follow-up of 180 days the patients had no serious complications observed during or after surgery. The relapse rate was $35.8 \%$ and occurred in primary $(35.7 \%)$ and recurrent $(36.4 \%)$ lesions with no statistical difference. Conclusions: This study suggests that intraoperative infiltration of 5-FU is safe. However the high recurrence rate indicated that other studies would be necessary to show the concentration/dose to better prevent it.

Keywords: Pterygium; Fluorouracil; Mitomycin/administration \& dosage; Chemotherapy adjuvant; Antimitotic agents; Evaluation of the efficacy-effectiveness of interventions; Intraoperative care; Recurrence/prevention \& control

\section{REFERÊNCIAS}

1. Duke-Elder S. Diseases of the outer eye. In: Duke-Elder S. System of Ophthalmology. St. Louis: Mosby; 1965. Chap.8. p.573.

2. Langham ME. The inhibition of corneal vascularization by triethylene thiophosphoramide. Am J Ophthalmol. 1960;49:1111-7.

3. Alves MR, Gaiotto Júnior OA, Kara-José N. Pterígio e tiotepa (relato de um caso com complicação grave irreversível). Arq Bras Oftalmol. 1981;44(5):164-6. 
4. Alves MR. Contribuição ao estudo dos efeitos locais do Tiotepa no pósoperatório do pterígio. Arq Bras Oftalmol. 1990;53(5):203-9.

5. Porto RB, Lacava AC, Falvo S, Centurion V. O uso da Mitomicina C tópica associada à exérese do pterígio. Rev Bras Oftalmol. 1999;58(2):135-40.

6. Frucht-Perry J, Ilsan M, Hemo I. Single dosage of mitomycin C for prevention of recurrent pterygium: preliminary report. Cornea. 1994;13(5):411-3.

7. Rubinfeld RS, Pfister RR, Stein RM, Foster CS, Martin NF, Stoleru S, Talley $\mathrm{AR}$, et al. Serious complications of topical mitomycin-C after pterygium surgery. Ophthalmology. 1992;99(11):1647-54. Commented in: Ophthalmology. 1992; 99(11):1645-6; Ophthalmology. 1993;100(7):977-7; author reply 977-8; Ophthalmology. 1993;100(7):976; author reply 977-8; Ophthalmology. 1993; 100(3):292-3.

8. Fujitani A, Hayasaka S, Shibuia Y, Noda S. Corneoscleral ulceration and corneal perforation after pterygium excision and topical mitomycin $\mathrm{C}$ therapy. Ophthalmologica. 1993;207(3):162-4.

9. Cardillo JA, Alves MR, Ambrosio LA, Poterio MB, Kara-Jose N. Single intraoperative application versus postoperative mitomycin $\mathrm{C}$ eyedrops in pterygium surgery. Ophthalmology. 1995;102(12):1549-52.

10. Alves MR. Perda de ceratócitos após desepitelização da córnea e uso tópico de mitomicina C. Rev Bras Oftalmol. 1996;55(3):268-75.

11. Susanna Jr R, Takahashi WY. Utilização de mitomicina em cirurgias combinadas. Rev Bras Oftalmol. 1996;55(1):13-5.

12. Yanyali AC, Talu H, Alp BN, Karabas L, Ay GM, Caglar Y. Intraoperative mitomycin $C$ in the treatment of pterygium. Cornea. 2000;19(4):471-3.

13. Cordova $\mathrm{H}$, Castellanos R. Evaluación in vitro de la viabilidad y proliferación de fibroblastos de la cápsula de Tenon humana: expuestos a 5-fluorouracilo y mitomicina C. Rev Oftalmol Venez. 1997;53(4):12-21.
14. Schellini SA, Shiratori CN, Spirandelli PH, Shiratori CA, Padovani CR. Uso do 5-fluorouracil no intraoperatório da cirurgia de pterígio. Arq Bras Oftalmol. 2000;63(2):111-4.

15. Shiratori CA, Hoyama E, Schellini SA, Padovani CR. Infiltração de 5-fluorouracil no pré-operatorio do pterígio. Arq Bras Oftalmol. 2003;66(4):499-503.

16. Norman S, Streiner A. Biostatistics: the bare essentials. St Louis: Mosby Year Book; 1994. 260p.

17. Akarsu C, Taner P, Ergin A. 5-Fluorouracil as chemoadjuvant for primary pterygium surgery: preliminary report. Cornea. 2003;22(6):522-6.

18. Maldonado MJ, Cano-Parra J, Navea-Tejerina A, Cisneros AL, Vila E, Menezo $\mathrm{JL}$. Inefficacy of low-dose intraoperative fluorouracil in the treatment of primary pterygium. Arch Ophthalmol. 1995;113(11):1356-7.

19. Evans RM, Laskin JD, Hakala MT. Assessment of growth-limiting events caused by 5 -fluorouracil in mouse cells and in human cells. Cancer Res. 1980; 40(11):4113-22.

20. Fernandes M, Sangwan VS, Bansal AK, Gangopadhyay N, Sridhar MS, Garg P, et al. Outcome of pterygium surgery: analysis over 14 years. Eye. 2005;19(11): $1182-90$.

21. Bekibele CO, Baiyeroju AM, Ajayi BG. 5-fluorouracil vs beta-irradiation in the prevention of pterygium recurrence. Int J Clin Pract. 2004;58(10):920-3.

22. Donnenfeld ED, Perry HD, Fromer S, Doshi S, Solomon R, Biser S. Subconjunctival mitomycin $\mathrm{C}$ as adjunctive therapy before pterygium excision. Ophthalmology. 2003;110(5):1012-6. Comment in: Ophthalmology. 2003;110(11): 2257-8; author reply 2258 .

23. Merriman MB, Mora JS, Beaumont BW, Merrilees MJ. Effects of varying 5fluorouracil exposure duration on tenon's capsule fibroblasts. Clin Exp Ophthalmol. 2001;29(4):248-52. 